ISSN 0103-8478

\title{
Eletroestimulação na cicatrização de feridas cutâneas experimentais em coelhos
}

\author{
Electrical stimulation in experimental wound healing in rabbits
}

\author{
Wanessa Krüger Beheregaray ${ }^{\mathrm{I}}$ Giordano Cabral Gianotti ${ }^{\mathrm{II}}$ Juliano de Souza Leal \\ Tuane Garcez ${ }^{\mathrm{II}}$ Emerson Antonio Contesini ${ }^{\mathrm{II}}$
}

RESUMO

Feridas cutâneas crônicas e complicadas são frequentemente encontradas na rotina da medicina veterinária $e$, muitas vezes, transformam os tratamentos em verdadeiro desafio. A eletroterapia tem sido indicada como alternativa aos tratamentos convencionais, devido à sua capacidade de promover cicatrização. O objetivo deste trabalho é relatar um ensaio experimental utilizando a eletroestimulação (ES) na cicatrização de feridas cutâneas em coelhos. Para tanto, foram utilizados 10 coelhos Nova Zelândia, machos, hígidos, submetidos à anestesia geral para a indução experimental de duas feridas cutâneas de $1 \mathrm{~cm}^{2}$ localizadas no dorso, caudal à borda das escápulas. As lesões foram higienizadas com solução salina $0,9 \%$, sendo a do lado direito tratada por ES e a do lado esquerdo, como controle. Para ES utilizou-se a frequência de $60 \mathrm{~Hz}$, por 10 minutos a cada dois dias, sendo aplicadas quatro agulhas de acupuntura em pontos equidistantes ao redor da lesão, a 0,5cm da borda. Os animais foram divididos em dois grupos, de oito e de 15 dias, conforme o período de tratamento e avaliação macroscópica e histopatológica. Não houve diferença significativa no tempo de cicatrização das lesões tratadas com ES. Porém, apresentaram qualidade tecidual, tanto na avaliação macro quanto microscópica, superior às do grupo controle. Com isso, é possível indicar a ES como tratamento de feridas cutâneas, por ser uma técnica eficiente, de fácil aplicação e de relativo baixo custo.

Palavras-chave: reparo cutâneo, eletroterapia, cicatriz.

\section{ABSTRACT}

Chronic skin wounds are frequently found in veterinary medicine routine and it becomes a challenge for clinicians. Sometimes the treatment routinely used end up failing. The electrotherapy has been indicated as an alternative to conventional treatments because of its ability to promote healing. The aim of this paper is to report an experimental study using electrical stimulation (ES) in wound healing in rabbits. It was evaluated 10 healthy New Zealand rabbits that were submitted to general anesthesia for resection of two wounds with $1 \mathrm{~cm}^{2}$ located in both sides of the back behind the scapula edge. It was determinate that the right wound would be treated by ES and the left would be used as a control, both cleaned with solution of $\mathrm{NaCl}$ $0,9 \%$. The frequency used to ES was $60 \mathrm{~Hz}$ for 10 minutes every two days, being applied by acupuncture needles in four equidistant points around the lesion, to $0.5 \mathrm{~cm}$ from the edge. The animals were divided in one group of 15 days and another one of 8 days for treatment and macroscopic and histopathological evaluation. The results showed that the lesions treated by ES do not have significant difference in healing time. However, those treated had higher scarring macroscopic and microscopic quality than the control group. Therefore, it is possible to indicate the use of ES as a skin wounds treatment, whereas it was demonstrated to be an effective technique, easy to use and has relatively low cost.

Key words: skin repair, electrotherapy, scar.

\section{INTRODUÇÃO}

Ferida é uma lesão caracterizada pela ruptura da continuidade normal da estrutura do corpo (WALDRON \& TREVOR, 1998). O reparo tissular é um processo complexo, que envolve a interação entre células estromais e circulatórias que são ativadas por uma plêiade de mediadores de natureza química diversificada, fragmentos de células e matriz extracelular, microorganismos e por alterações físicoquímicas no microambiente da lesão e das áreas a ela circunjacentes (BALBINO et al., 2005; PAVLETIC,

\footnotetext{
${ }^{\text {I }}$ Programa de Pós-graduação em Ciências Veterinárias, Universidade Federal do Rio Grande do Sul (UFRGS), Av. Bento Gonçalves, 9090 , Bairro Agronomia, 90540-000, Porto Alegre, RS, Brasil. E-mail: wbeherega@gmail.com. Autor para correspondência.

II Programa de Pós-graduação em Ciências Veterinárias, UFRGS, Porto Alegre, RS, Brasil.

${ }^{\text {III }}$ Faculdade de Veterinária, UFRGS, Porto Alegre, RS, Brasil. Recebido 18.01.12 Aprovado 06.06.13 Devolvido pelo autor 12.03.14 
2010). A possibilidade de acelerar a cicatrização e o fechamento de lesões cutâneas mediante recursos químico-medicamentosos ou físicos tem sido objeto de investigação de inúmeros pesquisadores (DORNELES, et al., 2003; GARROS et al., 2006; GURTNER et al., 2008).

Vários estudos têm demonstrado efeitos benéficos da eletroestimulação (ES) sobre processo cicatricial, indicada no tratamento tanto de condições agudas, quanto crônicas (ABOLAFIA et al., 1985; REICH \& TARJAN, 1990; CASAUBÓN \& LÓPEZ, 1991; SCOGNAMILLO-SZABÓ \& BECHARA, 2001; SUMANO et al., 2002; FREITAS et al., 2006; BEHEREGARAY et al., 2009).

A ES age incrementando o processo inflamatório agudo inicial, provavelmente acelerando o processo de eliminação dos detritos celulares e bacterianos, por meio da ativação de mediadores químicos responsáveis pela quimiotaxia de neutrófilos, que junto com os macrófagos fazem a fagocitose (ABOLAFIA et al., 1985; CASAUBÓN \& LÓPEZ, 1991; SUMANO et al., 2002). Além disso, estudos demonstram que a ES pode aumentar o fluxo sanguíneo e incrementar os fatores de crescimento e alinhamento das fibras de colágeno (JANSEN et al., 1989; SUMANO et al., 2002). Dessa forma, o objetivo deste trabalho é avaliar o efeito da eletroestimulação na cicatrização de feridas experimentais em coelhos.

\section{MATERIAL E MÉTODOS}

Foram avaliados 10 coelhos machos, hígidos, da raça Nova Zelândia, de origem controlada, peso de 3,5 a 4,5kg e idade variando de 120 a 160 dias. Os animais foram submetidos à anestesia geral para a realização das feridas. Para tanto, foi utilizada como medicação pré-anestésica cloridrato de quetamina $8 \mathrm{mg} \mathrm{kg}^{-1}$, IM, midazolam $0,8 \mathrm{mg} \mathrm{kg}^{-1}$, IM e citrato de fentanila $0,015 \mathrm{mg} \mathrm{kg}^{-1}$, IM, indução e manutenção anestésica com isoflurano vaporizado em $\mathrm{O}_{2}, 100 \%$. Após antissepsia, foi realizada a remoção de um retalho de pele e tecido subcutâneo de $1 \mathrm{~cm}^{2}$ do lado direito e outro do esquerdo, caudal à borda da escapula, a $1 \mathrm{~cm}$ da coluna vertebral. No pós-operatório imediato, as feridas foram medidas com paquímetro, cobertas por gaze estéril e mantidas com atadura elástica (Figura 1A). Administrou-se cloridrato de tramadol $3 \mathrm{mg} \mathrm{kg}^{-1}$, IM, no pós-operatório, BID, por três dias.

Transcorridas 24 horas da indução de cada lesão, realizava-se a mensuração do comprimento e da largura, com auxílio de paquímetro, seguida pela limpeza de ambas as lesões, com solução de $\mathrm{NaCl} 0,9 \%$, e então iniciava-se o tratamento com
ES nas lesões localizadas do lado direito. As lesões do lado esquerdo representavam o controle, isto é, desempenhavam apenas o papel de elemento de comparação e, para tanto, o único tratamento que recebiam diariamente era a limpeza com solução de $\mathrm{NaCl} 0,9 \%$.

As agulhas de acupuntura de $0,25 \times 15 \mathrm{~mm}$ foram aplicadas em quatro pontos equidistantes ao redor da lesão tratada, a uma distância de $0,5 \mathrm{~cm}$ de sua margem. Logo em seguida, o aparelho de ES (Sikuro, DSC 100) foi conectado às agulhas, a uma frequência de $60 \mathrm{~Hz}$, pulso alternado assimétrico intermitente e corrente alternada, variando de 8 a $20 \mathrm{~mA}$ (dependendo da resistência do animal), durante 10 minutos, a cada dois dias, por um período de 15 dias no grupo A e oito no B. Os animais, após o início do tratamento, permaneceram sem curativos e as lesões foram higienizadas duas vezes ao dia com solução salina isotônica.

As avaliações macroscópicas das feridas foram feitas segundo BEHEREGARAY et al. (2010), sendo que, em cada avaliação, as feridas foram mensuradas em duas direções, o maior comprimento pela menor largura, com auxílio de paquímetro digital. A área de contração da ferida (C) foi calculada subtraindo-se a área inicial (a1), determinada no dia da indução da lesão, da área da lesão (a) nos dias 2, $4,6,8,10,12,14,15$ para o grupo A, e nos dias 2 , 4, 6, 8 para o grupo B. Assim, C = a1 - a. A taxa de cicatrização (Tc) também foi avaliada a partir desses resultados $(\mathrm{Tc}=\mathrm{c} \times 100 / \mathrm{a} 1)$. Ainda foram feitos registros fotográficos durante as mensurações e os aspectos macroscópicos, como presença de tecido necrótico, granulação, sinais de infecção, presença de exsudato, hiperemia, edema, cianose e crosta, os quais, quando observados, foram anotados em fichas controle. Para a avaliação histológica da qualidade da cicatrização, por meio de exame de microscopia óptica, foram coletadas biópsias das feridas, através do uso de punch de $6 \mathrm{~mm}$, das lesões da direita e esquerda, no décimo quinto dia no grupo $\mathrm{A}$, e no oitavo dia no grupo B.

As amostras coletadas permaneceram conservadas por 48 horas em formol tamponado $10 \%$. A seguir, foram inclusas em parafina, sendo então submetidas, com micrótomo, a cortes transversais de $4 \mu \mathrm{m}$. Foram coradas com hematoxilina-eosina (HE), para avaliação global dos cortes de tecido, e com Tricômico de Masson (TM), para avaliar a presença de fibras colágenas existentes nos locais de fibrose do interstício. As variáveis histológicas avaliadas, conforme descrito por BEHEREGARAY et al. (2010), foram: proliferação vascular, células 


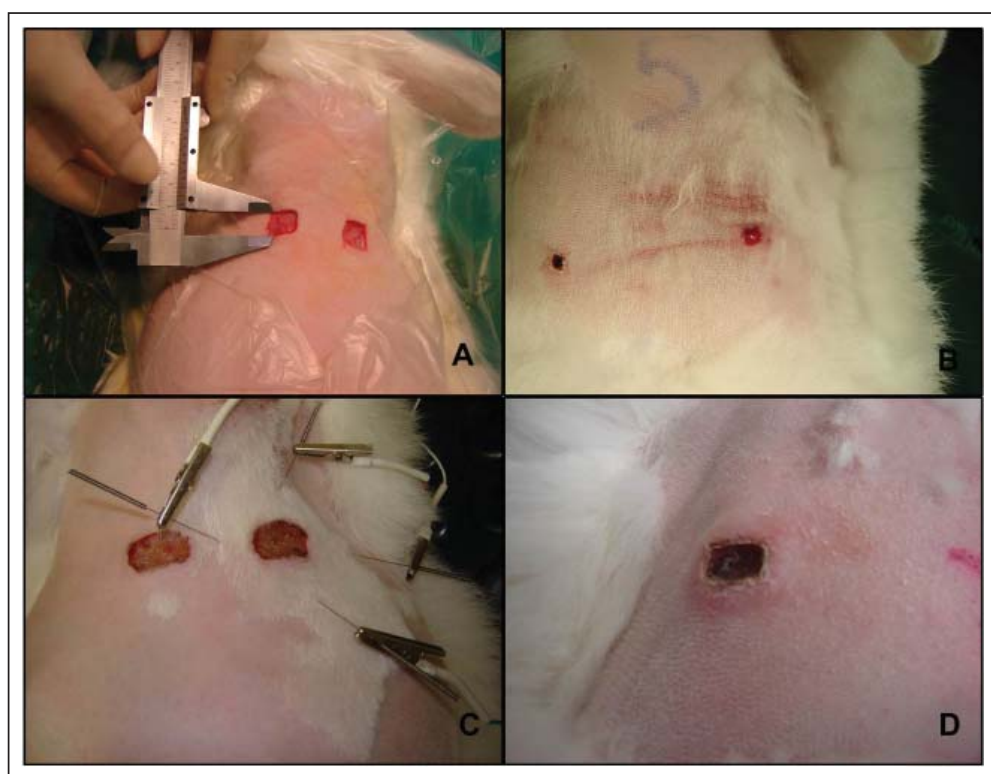

Figura 1 - (A) Imagem obtida no pós-operatório imediato da indução cirúrgica de lesões cutâneas em coelhos, demonstra a mensuração das feridas por meio do uso de paquímetro. (B) Aspecto vermelho brilhante da lesão da direita tratada por eletroestimulação (ES) em comparação com a lesão controle. (C) Imagem obtida durante o tratamento com ES, demonstrando o posicionamento das agulhas de acupuntura ao redor da lesão conectadas aos cabos do aparelho de ES. Além disso, também é possível visualizar um rápido crescimento piloso ao redor da ferida tratada por ES, quando comparada com a controle. (D) Por meio dessa imagem, é possível visualizar que a lesão controle apresentavase rodeada por um halo de pele dura ao tato, presença de crosta espessa recobrindo a ferida na quarta avaliação.

mononucleares e polimorfonucleares, proliferação fibroblástica, colagenização, reepitelização, presença de folículos pilosos e queratina. Foram atribuídos índices histológicos para os achados: ausente, igual a zero; discreto, igual a um; moderado, igual a dois; intenso, igual a três (Tabela 1).
A avaliação estatística dos dados foi feita pelo programa Statistical Package for the Social Sciences (SPSS). As variáveis histológicas foram analisadas mediante testes não-paramétricos de Mann-Whitney e de Wilcoxon. O nível de significância utilizado para rejeitar a hipótese de nulidade foi de $5 \%(\mathrm{P}<0,05)$.

Tabela 1 - O índice histológico utilizado como critério para avaliação da cicatrização de feridas cutâneas induzidas em coelhos, com valores variando entre 0 e 3, baseou-se em valores de proliferação vascular, células mononucleares, células polimorfonucleares, proliferação fibroblástica, colagenização, re-epitelização, folículos pilosos, queratina.

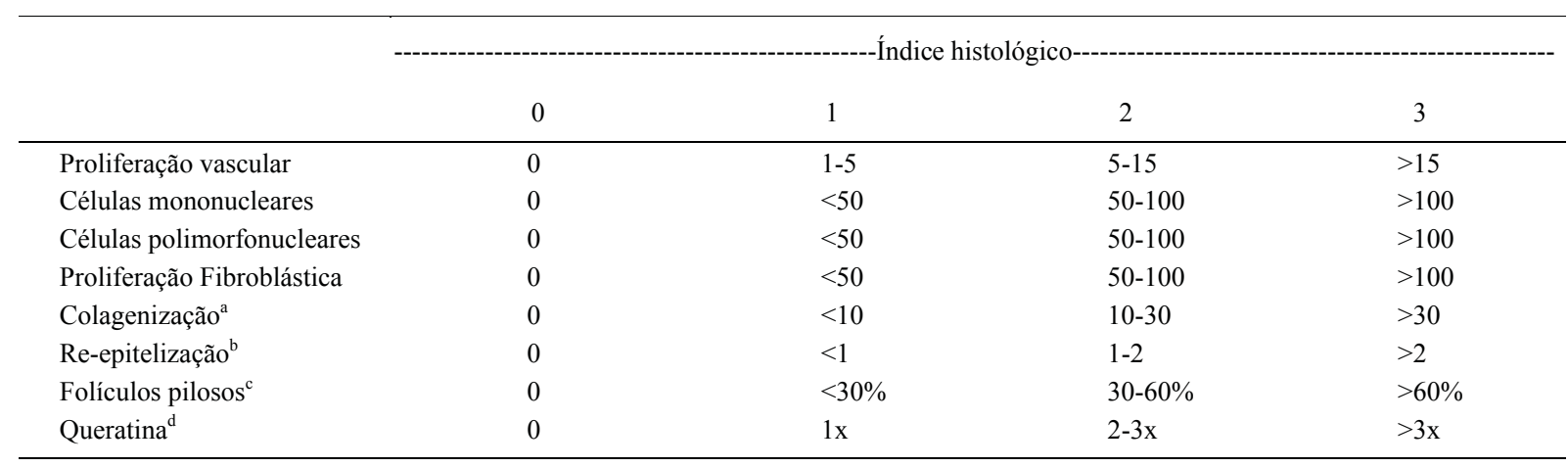

avaliação feita na região sublesional; ${ }^{b}$ relação feita através da visualização de um campo de $400 x$; ${ }^{c}$ porcentagem estabelecida em relação ao tamanho do bulbo do folículo piloso; ' proporção feita em relação ao tamanho da lâmina basal do epitélio.

Ciência Rural, v.44, n.5, mai, 2014. 


\section{RESULTADOS E DISCUSSÃO}

O procedimento cirúrgico para a indução das lesões transcorreu sem complicações em todos os animais. A recuperação anestésica e a analgesia pósoperatória mostraram-se satisfatórias. A metodologia aplicada na indução das lesões foi baseada em experimento prévio (BEHEREGARAY et al., 2010).

A medicação utilizada, pertencente à classe dos opioides, parece ter sido eficiente em promover analgesia, sem, contudo, apresentar características como ação anti-inflamatória, o que poderia alterar os resultados do experimento, visto que a inflamação constitui parte do processo de cicatrização (CASAUBÓN \& LÓPEZ, 1991; SUMANO et al., 2002; BALBINO et al., 2005; PAVLETIC, 2010). No entanto, nessa espécie, é particularmente difícil determinar o grau de dor; por ser um desafio, a literatura nessa área é restrita. WEVER et al. (2010), em um estudo de avaliação de dor pós-operatória em coelhos, comprovaram esse fato, pois encontraram resultados semelhantes em animais tratados e controles, não sendo possível determinar o melhor tratamento a ser empregado.

Os animais apresentaram-se dóceis na manipulação, aceitando o tratamento por ES sem necessidade de qualquer medicação para contenção e aplicação da técnica. Esses permaneciam relaxados no período de tratamento, podendo-se, por isso, supor que a sensação proporcionada pela ES é agradável. O mesmo comportamento foi constatado por BEHEREGARAY et al. (2009), utilizando a ES na cicatrização de lesão cutânea de um felino.

As agulhas de acupuntura foram escolhidas para transmitir a corrente elétrica, porque podem ser colocadas e removidas suavemente da pele saudável que rodeia a ferida, causando lesão tecidual mínima (SCHOEN, 2006). Seu comprimento permite a formação de um circuito fechado ao redor da lesão, o qual foi recomendado no estudo de SUMANO et al. (2002). Com a utilização de agulhas, os fluidos internos agem como um condutor natural, espalhando o estímulo elétrico em toda lesão, evitando o que ocorre quando se usa eletrodos adesivos, que cobririam toda a região da ferida. Ainda, esses adesivos não são aplicáveis em superfícies irregulares (SUMANO et al., 2002; LOWDEN \& REED, 2003).

A frequência de $60 \mathrm{~Hz}$ utilizada para ES foi determinada com base em outros trabalhos citados na literatura (ABOLAFIA et al., 1985; CAUSABÓN \& LOPEZ, 1991; SUMANO et al., 2002), diferenciando-se pela forma intermitente de estimulação e pelo pulso ser alternado simétrico, o que dificulta a acomodação do estímulo. Existem diferentes modos de aplicar a ES; correntes entre 20 e $100 \mathrm{~Hz}$, alternadas ou diretas, têm sido indicadas para estimular a cicatrização de feridas, devido ao incremento dos fatores de crescimento e pelo alinhamento das fibras de colágeno (SUMANO et al., 2002). É importante ressaltar que a intensidade da corrente aplicada sobre a lesão deve ser menor do que a que causa contração muscular (LOWDEN \& REED, 2003).

Desde a primeira aplicação da ES até o final do tratamento, pôde-se observar hiperemia ao redor da ferida durante o estímulo elétrico, provavelmente, devido ao aumento do aporte sanguíneo no local (Figura 1B). Outros autores observaram hiperemia durante o tratamento com ES e verificaram que isso ocorreria devido ao aumento da irrigação sanguínea (ABOLAFIA et al., 1985; CAUSABÓN \& LOPEZ, 1991; SUMANO et al., 2002; BEHEREGARAY et al., 2009). Ainda, JANSEN et al. (1989) sugeriram duas possíveis vias para ES aumentar o fluxo sanguíneo: uma inibição de fibras nervosas vasoconstrictoras simpáticas ou a liberação de neurotransmissores nas terminações nervosas periféricas de neurônios sensitivos primários de pequeno diâmetro (fibras $\mathrm{C}$ e $\mathrm{A} \delta$ ).

As lesões experimentais, de uma maneira geral, não apresentavam exsudação. Apenas um dos animais apresentou contaminação das feridas, com presença de exsudato purulento após quatro dias da indução da lesão. Somente nesse caso optou-se pela instituição da antibioticoterapia com o uso de enrofloxacina $10 \mathrm{mg} \mathrm{kg}^{-1}$ SID por cinco dias. Tendo sido observado, nesse caso, que a ferida tratada por ES apresentou remissão dos sinais de contaminação mais rapidamente que a controle, logo após a segunda aplicação da ES. A antibioticoterapia só foi mantida devido à resposta lenta da ferida controle. A diferença da resposta da ferida tratada com ES em relação à controle pode ser atribuída ao incremento do processo inflamatório agudo inicial, proporcionado pela ES. Esse processo acelera a eliminação dos detritos celulares e bacterianos, por meio da ativação de mediadores químicos responsáveis pela quimiotaxia de neutrófilos que, junto com os macrófagos, fazem a fagocitose (ABOLAFIA et al., 1985; CASAUBÓN \& LÓPEZ, 1991; SUMANO et al., 2002). Os dados desse animal foram retirados do experimento, pois o tempo cicatricial foi alterado e isso aumentaria ainda mais a variabilidade dos resultados no ponto de vista estatístico.

Em dois dos coelhos, pôde-se observar um crescimento piloso mais rápido ao redor da ferida tratada por ES, sendo necessário frequentes tricotomias para facilitar a higienização da ferida. Isso ocorreu, provavelmente, porque a cicatrização acelerada também produz estímulos para a formação 
de novos folículos pilosos, proporcionando o crescimento de pelos (Figura 1C). Essa hipótese pôde ser confirmada pelo exame microscópico, que evidenciou grande quantidade de folículos pilosos e glândulas na derme das lesões tratadas por ES. Porém, quando comparadas às médias do grupo A nos tratados $(2,40)$ e controles $(1,40)$, não houve diferença estatística significativa $(\mathrm{P}=0,197)$ para a presença de folículos pilosos. No grupo B, não houve presença de folículos pilosos, como descritos na tabela 2, devido à imaturidade da cicatriz de oito dias.

$\mathrm{Na}$ avaliação da área de cicatrização das lesões, não foi possível observar diferença na taxa de cicatrização dia-a-dia do grupo A (15 dias), quando comparados aos tratados pela ES com os controles. A taxa de cicatrização dia-a-dia do grupo B (oito dias) demonstra comportamento semelhante ao grupo A, não havendo diferenças entre tratados e controles.

A região em torno das feridas tratadas por ES apresentava textura da pele adjacente à lesão semelhante à pele normal. Já nas imediações da ferida controle, a pele apresentava-se rodeada por um halo de pele firme ao tato e aspecto áspero (Figura 1D). Podemos atribuir essa diferença entre tratados e controles ao fato de a ES promover o depósito e o alinhamento das fibras de colágeno. Isso pôde ser confirmado com o exame histopatológico, que mostra a deposição de colágeno estatisticamente superior $(\mathrm{P}=0,046)$ na tratada em comparação com o controle no grupo $\mathrm{A}$. No grupo $\mathrm{B}$, não houve diferença significativa entre tratado e controle (Tabela 2). A deposição de colágeno é feita, a princípio, de maneira aleatória, tendo como orientação a organização da fibronectina e dependente da natureza e direção das tensões aplicadas ao tecido. Repetições sucessivas da lise, síntese, redirecionamento e ligação formam fibras maiores de colágeno e resultam numa configuração mais regular da cicatriz. Isso aumenta a sua resistência, devido à organização das fibras acompanharem as forças mecânicas a que o tecido está sujeito durante a atividade normal (BALBINO et al., 2005). Provavelmente, a ES age de maneira benéfica, acelerando esse processo de um modo que resulta na diferença da textura da pele entre as tratadas e controles.

Os resultados encontrados neste experimento foram semelhantes aos descritos por CASAUBÓN \& LÓPEZ (1991), cabendo, entretanto, notar que estes consideraram que a cicatrização por ES ocorreu por regeneração e não por reparação, devido à semelhança histopatológica que havia com a pele normal. Com isso, CASAUBÓN \& LÓPEZ (1991) concluíram que pode existir um mecanismo celular ativado pela eletricidade para promover a síntese de colágeno, e outro que permita orientar as fibras, de maneira estratégica, para aumentar a força de contração das bordas da ferida.

No grupo B (oito dias), o exame microscópico evidenciou diferenças na espessura do epitélio, bem como na organização do tecido conjuntivo das lesões tratadas por ES e as controles. No grupo A, a diferença entre a ferida tratada e a controle foram mínimas. Provavelmente, isso ocorreu devido a ES ter seu efeito mais evidente na reepitelização numa fase mais precoce da cicatrização. Contudo, essas diferenças não foram estaticamente

Tabela 2 - Valores médios dos achados histopatológicos dos animais tratados com eletroestimulação ou controle durante 15 dias (grupo A ) e dos animais tratados com eletroestimulação ou controle durante 08 dias (grupo B). O índice histológico utilizado como critério para avaliação da cicatrização de feridas com valores variando entre 0 e 3 baseou-se em: proliferação vascular, células mononucleares, células polimorfonucleares, proliferação fibroblástica, colagenização, re-epitelização, folículos pilosos e queratina.

\begin{tabular}{lccccc}
\hline & Grupo A Tratada & Grupo A Controle & Valor P & Grupo B Tratada & Grupo B Controle \\
\hline Proliferação vascular & 2,8 & 3,0 & 0,13 & 2,0 & 1,75 \\
Células mononucleares & 1,8 & 1,6 & 0,705 & 1,5 & 1,5 \\
Células Polimorfonucleares & 1,0 & 2,00 & 0,059 & 1,5 & 1,313 \\
Proliferação Fibroblástica $^{\text {Colagenização }}{ }^{\mathrm{a}}$ & 2,6 & 2,2 & 0,317 & 2,0 & 1,5 \\
Re-epitelização $^{\mathrm{b}}$ & 2,8 & 2,0 & $0,046^{*}$ & 1,25 & 1,25 \\
Folículos pilosos $^{\mathrm{c}}$ & 2,8 & 2,2 & 0,257 & 0,25 & 1,0 \\
Queratina $^{\mathrm{d}}$ & 2,4 & 1,4 & 0,197 & 0 & 0 \\
\hline
\end{tabular}

a avaliação feita na região sublesional; ${ }^{b}$ relação feita através da visualização de um campo de 400x; ${ }^{c}$ porcentagem estabelecida em relação ao tamanho do bulbo do folículo piloso; ${ }^{\mathrm{d}}$ proporção feita em relação ao tamanho da lâmina basal do epitélio.

*Diferença estatística significativa $(\mathrm{P}<0,05)$ usando o teste Mann-Whitney. 
significativas para nenhum dos itens avaliados. Uma hipótese para tal resultado seria uma possível interferência da ferida tratada sobre a controle, já que ambas eram realizadas no mesmo animal. Desse modo, a metodologia proposta neste trabalho objetivou minimizar a interferência que ocorre na cicatrização entre indivíduos e desconsiderou a possibilidade de haver interferência do tratamento local poder interferir de maneira sistêmica na ferida controle. Para tanto, é necessário que sejam realizados novos estudos, utilizando indivíduos diferentes para tratamento e controle.

Os indícios encontrados nesse experimento indicam efeito benéfico desse protocolo (frequência da corrente, tempo de estímulo e periodicidade) de ES sobre a cicatrização; bem como a forma de aplicação é fácil, relativamente econômica e aparentemente eficaz.

\section{CONCLUSÃO}

Com a realização deste estudo foi possível concluir que as lesões tratadas com ES apresentavam qualidade cicatricial, a partir da avaliação macroscópica, superiores às controles. Pode-se atribuir o efeito cosmético favorável dessa técnica, provavelmente, à quantidade superior de colágeno presente na lesão tratada, já que esse elemento é um dos principais responsáveis pela qualidade e resistência de uma cicatriz.

\section{COMITÊ DE ÉTICA}

Este projeto foi avaliado e aceito pelo Comitê de ética do Hospital de Clínicas de Porto Alegre, sob o registro: Protocolo $\mathrm{n}^{\mathrm{o}}$ 10-0111.

\section{REFERÊNCIA}

ABOLAFIA, A.J.A. et al. Evaluación del efecto cicatrizante de la acupuntura. Veterinaria Mexico, v.16, p.27-31, 1985.

BALBINO, C.A. et al. Mecanismos envolvidos na cicatrização: uma revisão. Revista Brasileira de Ciências Farmacológicas, v.41, n.1, p.27-51, 2005. Disponível em: <http://www.scielo. br/scielo.php? pid $=$ S 1516-93322005000100004\&script $=$ sci arttext>. Acesso em: 5 maio, 2013. doi: 10.1590/S151693322005000100004

BEHEREGARAY, W.K. et al. Tratamento de ferida por eletroacupuntura em uma gata. Acta Scientiae Veterinariae, v.37, n.3, p.285-289, 2009. Disponível em: <http://www.ufrgs.br/ actavet/37-3/PUB\%20845.pdf>. Acesso em: 5 maio, 2013.

BEHEREGARAY, W.K. et al. Uso do Laser ALGaInP na cicatrização de lesões cutâneas experimentais em coelhos. Acta Scientiae Veterinariae, v.38, n.3, p.237-243, 2010. Disponível em: <http://www.ufrgs.br/actavet/38-3/904.pdf>. Acesso em: 05 maio, 2013.
CASAUBÓN, T.; LÓPEZ, H.S. Efecto de la eletroestimulacións sobre heridas que cicatrizan por segunda intención en ratas. Veterinária Mexico, v.22, n.4, p.284-289, 1991.

DORNELES, D. et al. Efeito de Aloe vera Linné sobre a cicatrização de feridas de pele em coelhos. Visão Acadêmica, v.4, n.1, p.39-46, 2003. Disponível em: < http://ojs.c3sl.ufpr.br/ojs2/ index.php/academica/article/viewArticle/521>. Acesso em: 05 maio, 2013.

FREITAS, P.M.C. et al. Eletroacupuntura aplicada nas fases precoce e tardia da cicatrização do tendão calcanear comum de coelhos após reparo tardio com peritônio bovino conservado em solução supersaturada de sal: aspectos clínicos. Ciência Rural, v.36, n.6, p.1830-1836, 2006. Disponível em: <http://www.scielo. br/pdf/cr/v36n6/a25v36n6.pdf>. Acesso em: 05 mai. 2013. doi: $10.1590 / \mathrm{S} 0103-84782005000200024$.

GARROS I.C. et al. Extrato de Passiflora edulis na cicatrização de feridas cutâneas abertas em ratos: estudo morfológico e histológico. Acta Cirúrgica Brasileira, v.21, n.3, p.5-65, 2006. Disponível em: <http://www.scielo.br/pdf/acb/v21s3/09.pdf>. Acesso em: 05 maio, 2013. doi: 10.1590/S0102-86502006000900009.

GURTNER, G.C. et al. Wound repair and regeneration. Nature, v.453, n.15, p.314-321, 2008. Disponível em: < http://www.nature. $\mathrm{com} /$ nature/journal/v453/n7193/full/nature07039.html>. Acesso em: 8 jun. 2008. doi: 10.1038/nature07039.

JANSEN, G. et al. Acupuncture and sensory neuropeptides increase cutaneous blood flow in rats. Neuroscience Letters, v.97, p.305-309, 1989.

LOWDEN, J.; REED, A. Eletroterapia explicada: princípios e prática. 3.ed. São Paulo: Manole, 2003. 471p.

PAVLETIC, M.M. Atlas of small animal reconstructive surgery. 3.ed. Iowa: Saunders, 2010. 680p.

REICH J.D.; TARJAN P.P. Electrical stimulation skin. International Journal of Dermatology, v.29, n.6, p.395400, 1990. Disponível em: <http://onlinelibrary.wiley.com/ doi/10.1111/j.1365-4362.1990.tb03820.x/abstract>. Acesso em: 05 maio, 2013. doi: 10.1111/j.1365-4362.1990.tb03820.x.

SCHOEN, A.M. Acupuntura veterinária: da arte antiga à medicina moderna. São Paulo: Roca, 2006. 603p.

SCOGNAMILLO-SZABÓ, M.V.; BECHARA, G.H. Acupuntura: bases cientificas e aplicações. Ciência Rural, v.31, n.6, p.10911099, 2001. Disponível em: <http://www.scielo.br/scielo. php? script $=$ sci_arttext\&pid $=$ S0103-84782001000600029\&ln$\mathrm{g}=\mathrm{en} \& \mathrm{nrm}=\mathrm{iso} \& \operatorname{lng}=\mathrm{pt}>$. Acesso em: 05 maio, 2013. doi: 10.1590/S0103-84782001000600029.

SUMANO, $\mathrm{H}$. et al. Use of electrical stimulation for wound healing in dogs. Disponível em: <http://www.isrvma.org/ article/57 1 7.htm>2002. Acesso em: abr. 2008.

WALDRON, D.R.; TREVOR, P. Tratamento dos ferimentos superficiais. In: SLATTER, D. Manual de cirurgia de pequenos animais. São Paulo: Manole, 1998. Cap.25, p.334-347.

WEAVER, L.A. et al. A model for clinical evaluation of perioperative analgesia in rabbits (Oryctolagus cuniculus). Journal of the American Association for Laboratory Animal Science, v.49, n.6, p.845-851, 2010. Disponível em: $<$ http://www.ncbi.nlm.nih.gov/pmc/articles/PMC2994053/pdf/ jaalas2010000845.pdf>. Acesso em: 05 maio, 2013. 